\title{
DERECHOEIECTORAL
}

\section{Participación en las elecciones municipales en Costa Rica: perspectiva histórica}

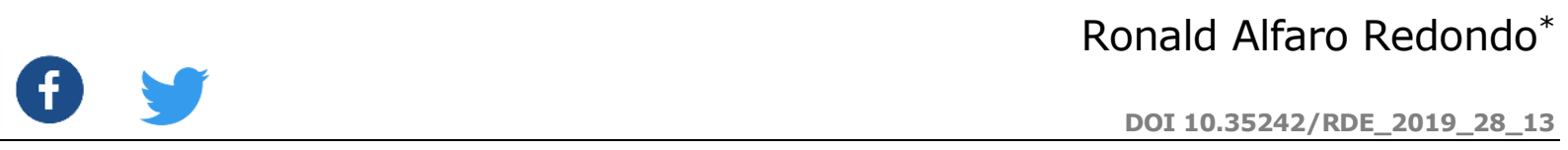

Nota del Consejo Editorial

Recepción: 15 de mayo de 2019.

Revisión, corrección y aprobación: 11 de junio de 2019.

Resumen: El artículo analiza los patrones de participación de los electores en las elecciones municipales del 2002 al 2016, para lo cual presenta una perspectiva histórica de los comicios y un análisis de la participación electoral por conglomerados y por comportamiento en el ejercicio del sufragio, revelando que el sector más participativo en estas elecciones se encuentra al este del país, mientras que, por otra parte, 4 de cada 10 votantes ha sufragado al menos en una de las tres elecciones celebradas.

Resumen: Participación política / Comportamiento del voto / Abstencionismo / Elecciones municipales.

Abstract: The article analyzes the patterns of voter participation in the municipal elections from 2002 to 2016, for which it presents a historical perspective of the elections and an analysis of electoral participation per conglomerate and per behavior when voting. It revealed that the most participative sector in these elections is the Eastern part of the country, whereas 4 out of every 10 voters have voted in at least one of three elections.

Key Words: Political participation / Behavior of suffrage / Abstentionism / Municipal elections.

\footnotetext{
* Costarricense, politólogo, correo ralfaro@estadonacion.or.cr. Investigador del Centro de Investigación y Estudios Políticos de la Universidad de Costa Rica y del Programa Estado de la Nación. Bachillerato y licenciatura en Ciencias Políticas, Universidad de Costa Rica. Máster en Métodos Cuantitativos en las Ciencias Sociales de la Universidad de Columbia en Nueva York, USA. Doctor en Ciencias Políticas de la Universidad de Pittsburgh, Pennsylvania, USA. Autor principal del estudio del Barómetro de las Américas para Costa Rica. Coordinador de la Unidad de Opinión Pública y Cultura Política del CIEP-UCR. Investigador del Programa Estado de la Nación. Profesor de la Escuela de Ciencias Politicas de la UCR. Sus áreas de trabajo son: política comparada, elecciones y sistemas electorales, partidos políticos; participación política, opinión pública y cultura política.
} 


\section{DERECHO ELECTORAL}

\section{INTRODUCCIÓN}

¿Por qué algunos ciudadanos votan y otros no? ha acaparado la atención de los investigadores $y$ ha originado una impresionante cantidad de contribuciones (Matsusaka y Palda, 1999). La participación electoral ha constituido uno de los temas que ha despertado mayor interés en la literatura sobre comportamiento político alrededor del mundo. Un interés similar ha despertado el debate acerca de si hay evidencia de una disminución de la participación en las democracias industrializadas y otros países en desarrollo (Nie et al., 1976; Powell, 1986; Franklin, 2004; Blais et al., 2004; Teixeira, 1987; Wattenberg, 1998; Lewis-Beck, 2008 y Flickinger y Studlar, 1992).

Algunos expertos han venido señalando que la conducta electoral es mejor comprendida si se analiza la persistencia de una elección a otra; es decir, el voto visto desde la perspectiva del hábito. Este abordaje se basa en la idea de que votar por primera vez en las elecciones en las que se tiene derecho a hacerlo deja una "huella" en el comportamiento político del individuo (Denny y Doyle, 2009). Esta persistencia acentúa la naturaleza dinámica del comportamiento electoral. Si el voto es un hábito, como la literatura señala, es esperable que la participación se incremente conforme los votantes tengan más oportunidades de participar, debido a que dicho hábito se refuerza con el paso del tiempo (Franklin, 2004 y Denny y Doyle, 2009).

Este artículo reformula la pregunta inicial y se plantea aportar respuestas a la interrogante de: ¿por qué algunos ciudadanos votan en elecciones locales? Al respecto, como parte de las explicaciones se ha mencionado que la participación en elecciones está determinada, en mayor medida que otras elecciones subterritoriales, por factores locales. Los autores han mencionado que aspectos como la cercanía a las instituciones locales, a los líderes municipales y el contacto más estrecho con las decisiones políticas son elementos que podrían favorecer la participación en los asuntos políticos locales. En este sentido, hay un reconocimiento explícito de que lo que se dilucida en elecciones municipales no son problemas nacionales, sino asuntos localizados en el entorno inmediato del elector (Delgado, 1998).

Por otra parte, las elecciones municipales poseen una particularidad propia: se encargan de materializar el principio de representación política en los municipios. Como es de esperar, las razones que están detrás de la participación política en el nivel municipal pueden ser muy variadas. Por ejemplo, según Navia (2003), pese a sus diferencias, las elecciones nacionales 


\section{DERECHO EIECTORAL}

y municipales se influencian mutuamente. Si un Gobierno nacional es relativamente bien evaluado por la opinión pública, se espera que los candidatos oficialistas obtengan buenos resultados en una elección municipal.

Del mismo modo, en buena parte de los países las elecciones municipales constituyen un termómetro para medir fuerzas y construir la maquinaria electoral de cara a una elección nacional. Incluso, en varios países los candidatos a presidentes son primero representantes municipales y luego aspiran a puestos nacionales.

En Latinoamérica, las elecciones subnacionales tienen una incidencia menor que las nacionales sobre la legitimidad y la gobernabilidad global del sistema político y, por ello, sería de esperar que en ellas los sistemas electorales adoptaran menores exigencias en cuanto a la primera y favorecieran la representatividad en mayor medida que los sistemas electorales nacionales (Molina, 2007).

Para los efectos de este trabajo, se considera la participación electoral como una modalidad de participación política especialmente importante, pues se trata de la forma de participación en la que más individuos intervienen a pesar de no ser necesariamente la más frecuente. Por ende, la reflexión en torno a las características de los participantes y su comparación con los no participantes es una de las cuestiones centrales en el análisis del comportamiento político (Anduiza y Bosch, 2004).

En este sentido, la participación política no es una actividad homogéneamente extendida entre los ciudadanos. Es decir, no todos los ciudadanos participan de igual forma ni con la misma frecuencia e intensidad (Anduiza y Bosch, 2004). Asimismo, la participación difiere según el tipo de elección. Hay elecciones que son más relevantes o trascendentales para los votantes que otras.

En el año 2002 los costarricenses eligieron por primera vez, de manera directa, a los alcaldes municipales, la principal autoridad política a nivel local. Junto con los alcaldes se nombraron los síndicos, cuya principal función es representar a los distritos de cada cantón. En 2002, 2006 y 2010 los concejales municipales, mejor conocidos como regidores, se eligieron junto con el presidente de la República y los diputados. Pero, a partir de 2016 se unificó la elección de todos los cargos municipales en una fecha separada de la elección presidencial y legislativa. 


\section{DERECHO ELECTORAL}

El artículo estudia en profundidad los patrones de participación de los votantes en los comicios municipales del período 2002-2016. Los dos hallazgos principales son, por un lado, que el análisis territorial de la participación electoral muestra un país dividido en dos: el este del país es menos participativo que el oeste del territorio. Por otra parte, un análisis longitudinal identificó que los votantes habituales en elecciones locales rondan el $10 \%$ de los habilitados para votar y que 4 de cada 10 votantes sufragó al menos en una de las tres elecciones celebradas entre 2002 y 2016.

\section{ELECCIONES MUNICIPALES EN PERSPECTIVA HISTÓRICA}

El domingo 7 de febrero de 2016 se realizaron en Costa Rica los cuartos comicios para elegir autoridades municipales en los 81 municipios y 480 distritos del país. En esta oportunidad se designaron un total de 6069 representantes políticos y compitieron más de 30000 candidatos. En la región latinoamericana Costa Rica fue uno de los últimos países en incorporar a su legislación la designación directa de este tipo de cargos (ver tabla 1 para más detalles). Si bien es cierto estas elecciones se desarrollaron en un ambiente de normalidad, predominó la baja participación y la apatía del electorado, dos atributos que han caracterizado la participación política a escala local en el período estudiado.

Los comicios se desarrollaron en un marco de respeto de los derechos civiles y políticos de todas las personas. Además, los resultados respondieron fielmente a la voluntad popular. No obstante, a pesar de un ligero incremento en la participación del $28 \%$ al $35 \%$ del padrón, predominó -una vez más- un elevado abstencionismo, que da cuenta de la enorme apatía de la ciudadanía para involucrarse en los asuntos municipales en un país que ha sobresalido históricamente por su fuerte centralismo estatal.

La baja participación en todo el país contrasta con las enormes disparidades de la participación en los municipios. Por ejemplo, en el municipio de mayor participación votó el $65 \%$ de los empadronados, equiparable a una elección nacional (Raventós et al., 2005) y en el de menor participación sufragó únicamente el $25 \%$. 


\section{DERECHOEIECTORAL}

SEGUNDO SEMESTRE 2019 NÚMERO 28

ISSN: 1659-2069

Tabla 1

Cifras relevantes de las elecciones municipales 2002-2016

\begin{tabular}{|c|c|c|c|c|}
\hline Indicador & 2002 & 2006 & 2010 & 2016 \\
\hline Padrón electorala/ & 2331459 & 2603770 & $\begin{array}{c}2865 \\
509\end{array}$ & 3178364 \\
\hline Juntas receptoras de votos & 6819 & 4852 & 5250 & 5630 \\
\hline Partidos políticos participantes ${ }^{\mathrm{b} /}$ & 34 & 43 & 47 & 59 \\
\hline Coaliciones ${ }^{c /}$ & & & 7 & 4 \\
\hline Cargos por elegird/ & 5758 & 5873 & 5952 & 6069 \\
\hline Candidaturas inscritas & 16261 & 15919 & 15858 & 31879 \\
\hline Alcaldes & 81 & 81 & 81 & 81 \\
\hline Vicealcaldes ${ }^{\mathrm{e} /}$ & 162 & 162 & 162 & 162 \\
\hline $\begin{array}{l}\text { Regidores } \\
\text { (propietarios y suplentes) } \text { f/ }^{2}\end{array}$ & 1002 & 1006 & 990 & 1010 \\
\hline $\begin{array}{l}\text { Síndicos } \\
\text { (propietarios y suplentes) }\end{array}$ & 913 & 924 & 927 & 960 \\
\hline $\begin{array}{lll}\text { Concejales } & \text { de } & \text { distrito } \\
\text { (propietarios y suplentes) } & \end{array}$ & 3528 & 3628 & 3712 & 3776 \\
\hline Intendentes & 8 & 8 & 8 & 8 \\
\hline $\begin{array}{l}\text { Concejales municipales de distrito } \\
\text { (propietarios y suplentes) }\end{array}$ & 64 & 64 & 64 & 64 \\
\hline Viceintendentes & & & 8 & 8 \\
\hline Participación electoral (\%) & 22,8 & 23,8 & 27,9 & 35,4 \\
\hline
\end{tabular}

Nota: Elaborado con base en los datos suministrados por el Tribunal Supremo de Elecciones.

a/ Por tratarse de elecciones locales, la cifra no incluye electores en el extranjero.

b/ Las cifras de 2010 y 2016 incluyen coaliciones.

c/ La figura existió a partir de las elecciones de 2010. Solo incluye uniones de dos o más partidos.

d/ En las elecciones de 2002, 2006 y 2010 se incluyen los cargos a nivel local de los comicios de febrero y diciembre de cada año.

e/ En las elecciones de 2002 y 2006 este cargo correspondía a alcaldes suplentes.

f/ En las elecciones de 2002, 2006 y 2010, los regidores no eran electos de forma simultánea al resto de cargos. Por este motivo, no se incluyen en el total de cargos por elegir.

Los resultados de las elecciones municipales del período 2002-2016 reflejan la consolidación del multipartidismo producto de un afianzamiento de los partidos locales (Alfaro, 2002; Alfaro y Gómez, 2014), el debilitamiento del Partido Liberación Nacional (PLN) y la apatía del electorado a pesar de un pequeño repunte en la participación. En lo que respecta a la concurrencia a 


\section{DERECHO ELECTORAL}

las urnas, se observa una baja participación del electorado (35\%), la cual concuerda con la tendencia observada en las tres anteriores elecciones (Alfaro, 2008).

\section{AUMENTA LA PARTICIPACIÓN EN ELECCIONES MUNICIPALES}

Tradicionalmente la baja participación en contiendas locales se atribuye al hecho de que dichos comicios son considerados como elecciones de segundo orden $\mathrm{y}$, por ende, de menor relevancia en perspectiva comparada (Heath et al., 1999; Müller, 1988; Trounstine, 2013 y Holbrook y Weinschenk, 2013). Costa Rica no es una excepción en este sentido.

En el caso costarricense, de los 3178364 electores habilitados para sufragar en 2016 , solo un $35 \%$ ejerció este derecho (siete puntos porcentuales más que en la última contienda en 2010). El ausentismo de votantes en las urnas fue alto en comparación con procesos similares en otros países y con el resto de elecciones nacionales, pero se mantuvo estable respecto de lo acontecido en 2002 , 2006 y 2010 (77\%, 77\% y 72\% respectivamente).

En los comicios municipales de 2016, una vez más los electores de localidades urbanas se abstuvieron de participar en mayor medida que los de comunidades rurales (Alfaro, 2002 y 2008). Los cantones cabecera de provincia (San José, Alajuela, Heredia, Cartago, Puntarenas, Liberia y Limón) encabezan los lugares con mayor ausentismo en las urnas en las respectivas provincias. En este grupo también se incluyen centros densamente poblados como Desamparados, Goicoechea, La Unión y Tibás. En estos cantones el abstencionismo superó el $70 \%$ de los electores como se observa en la figura 1.

Por otra parte, los cantones con menor abstencionismo son principalmente rurales, y entre ellos sobresalen Montes de Oro, San Mateo, Turrubares y Hojancha (localidades en las que alrededor del 65\% de los electores concurrieron a las urnas). Además, una parte importante de estos cantones pertenece a Guanacaste, Puntarenas y Limón que son las provincias con mayores niveles de abstencionismo en las elecciones presidenciales y legislativas.

Este patrón de comportamiento, que difiere de lo que sucede en las elecciones nacionales en las que predomina la división centro-periferia (Raventós et al., 2005), puede encontrar explicación en el hecho de que para las comunidades 


\section{DERECHO ELECTORAL}

rurales los Gobiernos locales revisten una mayor importancia y tienen mayor presencia institucional que las entidades del Gobierno central. El análisis de los patrones de abstencionismo revela importantes similitudes a lo largo del tiempo; es decir, las municipalidades que reportan los niveles más altos y más bajos de participación tienden a ser las mismas en las cuatro elecciones analizadas.

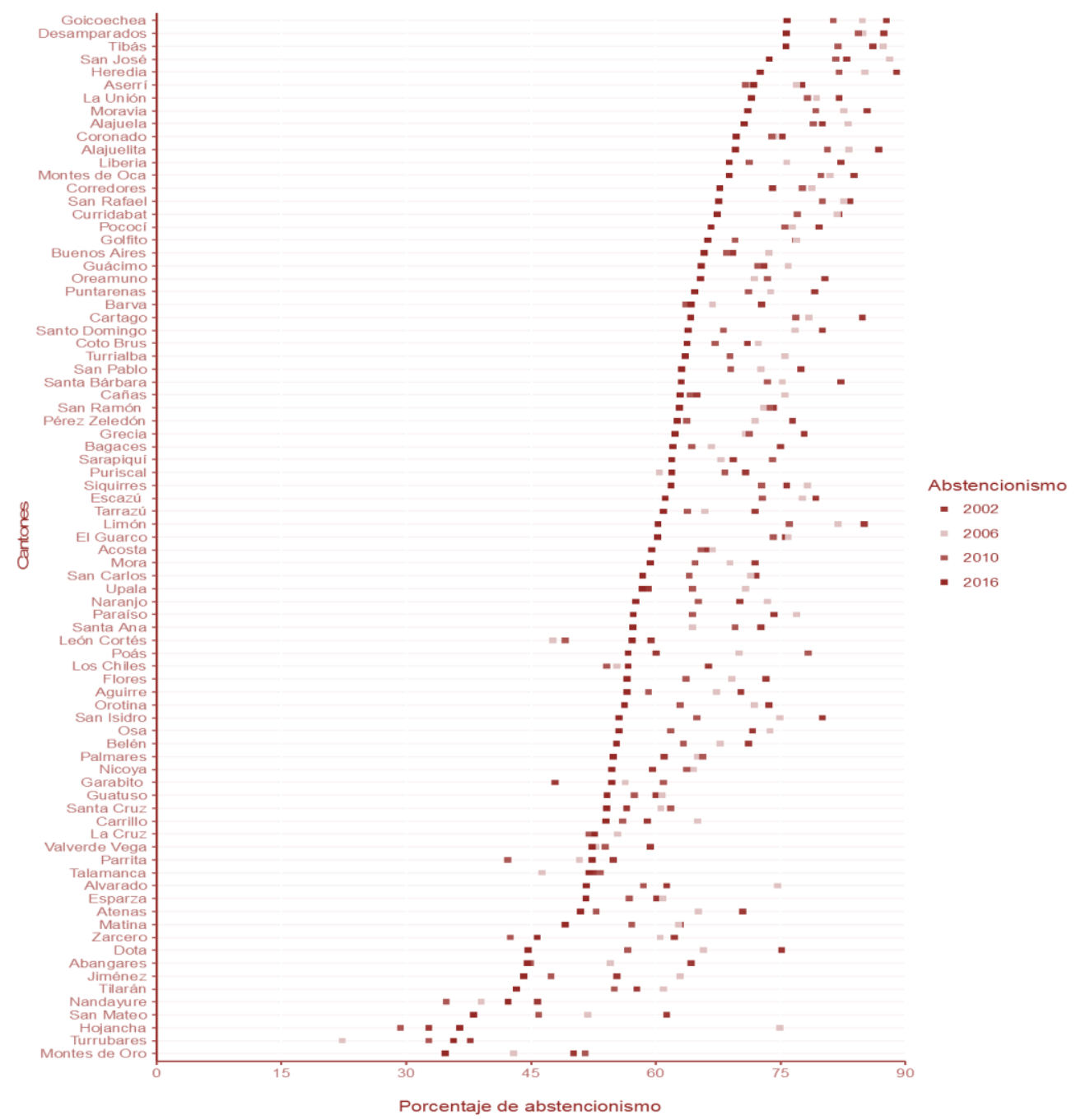

Figura 1. Abstencionismo en elecciones municipales, según cantón. 2002-2016 (porcentajes). Programa Estado de La Nación [PEN] (2011). 


\section{DERECHO ELECTORAL}

\section{ECOLOGÍA DE PARTICIPACIÓN PROPIA}

Desde los comicios municipales de 2002, la asistencia de los costarricenses a las urnas ha oscilado entre un $23 \%$ y un $35 \%$. Para un país cuya legislación no prevé sanciones a la abstención electoral, este nivel de participación es bajo. No obstante, ha venido aumentando con cada elección. Una de las consecuencias de este fenómeno, poco analizada hasta ahora, es el surgimiento de zonas de mayor o menor participación a escala municipal. En otras palabras, la movilización de los electores muestra diferencias significativas por región del país.

Las y los ciudadanos de esas regiones con mayor asistencia a las urnas están más involucrados en la competencia electoral, mientras que en las áreas restantes las personas están desmovilizadas de este proceso democrático básico, pues tienen una participación mínima. Vale decir que las ecologías del voto municipal son, además, muy distintas a las que se observan en las elecciones nacionales.

Para realizar el análisis de las ecologías territoriales del voto se recopiló la información electoral municipal del 2016, desagregada a nivel de juntas y centros de votación -el mayor nivel de desagregación posible- y se identificaron conglomerados de alta y baja participación electoral (recuadro 1).

\section{Recuadro 1: Análisis de conglomerados de participación electoral municipal en 2016}

Según Waldo Tobler (1970, p. 236), la primera ley de la geografía estipula que "todas las cosas están relacionadas entre sí, pero las cosas más próximas en el espacio tienen una relación mayor que las distantes". Desde esta perspectiva cabría esperar que, en materia de participación electoral local, los centros de votación cercanos geográficamente sean más similares entre sí, que con respecto a recintos más lejanos. Las características sociodemográficas y socioeconómicas de los pobladores de centros vecinos tenderían a ser bastante homogéneas, por lo que es altamente probable que sus patrones de votación en elecciones municipales también lo sean.

Este artículo explora ese supuesto con herramientas de análisis geográfico. La información base incluye la georreferenciación de cada uno de los centros de votación (unidad de análisis) en las elecciones municipales de 2016 y la participación electoral en cada junta receptora de votos instalada en esos recintos. Se utilizó el software ArcGis 10.5.

Se aplicó el índice de Moran I, que permite analizar conglomerados espaciales de alta y baja participación electoral a partir de tres valores. En primer lugar, se calcula la puntuación del índice. En seguida se estima una puntuación z (z-scores) basada en el promedio de participación de todas las juntas receptoras de votos del recinto electoral (escuela o colegio, 


\section{DERECHO EIECTORAL}

por ejemplo). En tercer lugar, se estima una probabilidad o valor de $p$ ( $p$-value), de que el patrón espacial observado se deba a un factor aleatorio. Además, se identifican valores atípicos que puedan sesgar o distorsionar los cálculos. La combinación de estos tres valores indica en qué medida la aparente similitud (en un conglomerado de altos o bajos valores) o diferencia (en el caso de los conglomerados de valores atípicos) es más pronunciada de la que podría esperarse en una distribución aleatoria.

Para interpretar los valores del índice se deben seguir los criterios que se mencionan a continuación. Un valor positivo indica que un centro de votación colinda con recintos con niveles similares o superiores de participación electoral, constituyendo así un conglomerado de alta participación. Por el contrario, un valor negativo indica que un centro de votación colinda con otros recintos con valores distintos (más bajos o más altos) de participación, conformando así un clúster de valores atípicos de concurrencia a las urnas. En ambos casos la probabilidad estimada debe ser lo suficientemente pequeña para que los clústeres de alta participación o de valores atípicos alcancen niveles de significancia estadística.

El resultado del análisis es un mapa en el que se distinguen cuatro tipos de conglomerados espaciales estadísticamente significativos al 0,05 de confianza: I) conglomerados de alta participación (alta-alta), II) conglomerados de baja participación (baja-baja), III) un primer conglomerado de valores atípicos en el cual un centro de alta participación colinda con recintos de baja participación (alto-bajo) y IV) un segundo conglomerado de valores atípicos en el cual un centro de baja participación colinda con recintos de alta participación (bajo-alto).

La figura 2 ilustra los hallazgos del estudio. Cada punto en el mapa representa un centro de votación (escuela, colegio u otro). Con este nivel de precisión es posible identificar las cuatro ecologías de participación electoral mencionadas y su localización geográfica, que dan cuenta de un sistema político local que configura un espacio de participación electoral heterogéneo. Estas ecologías locales configuran el mapa político local, en el cual se observa una clara división este-oeste que parte al país prácticamente en dos donde se distingue:

- Una ecología de alta participación en el oeste del territorio que cubre toda la provincia de Guanacaste, parte de Puntarenas y Alajuela, y pequeños conglomerados de juntas receptoras de votos con alta participación en Limón, Cartago, Heredia y San José o incluso en la zona sur (puntos rojos).

- Ecologías de muy baja participación en el este del país, fundamentalmente en el centro del territorio y en zonas costeras y limítrofes. Vale anotar que algunos de estos sitios (centros urbanos) concentran la mayor cantidad de población y, a la vez, son los que menos participan en política (puntos verdes). 


\section{DERECHO ELECTORAL}

- Otros sitios en los que no se identificaron patrones claros, o bien, se encontraron asociaciones que no son estadísticamente significativas (puntos azules y puntos amarillos), por lo general mezcladas en zonas de baja participación.

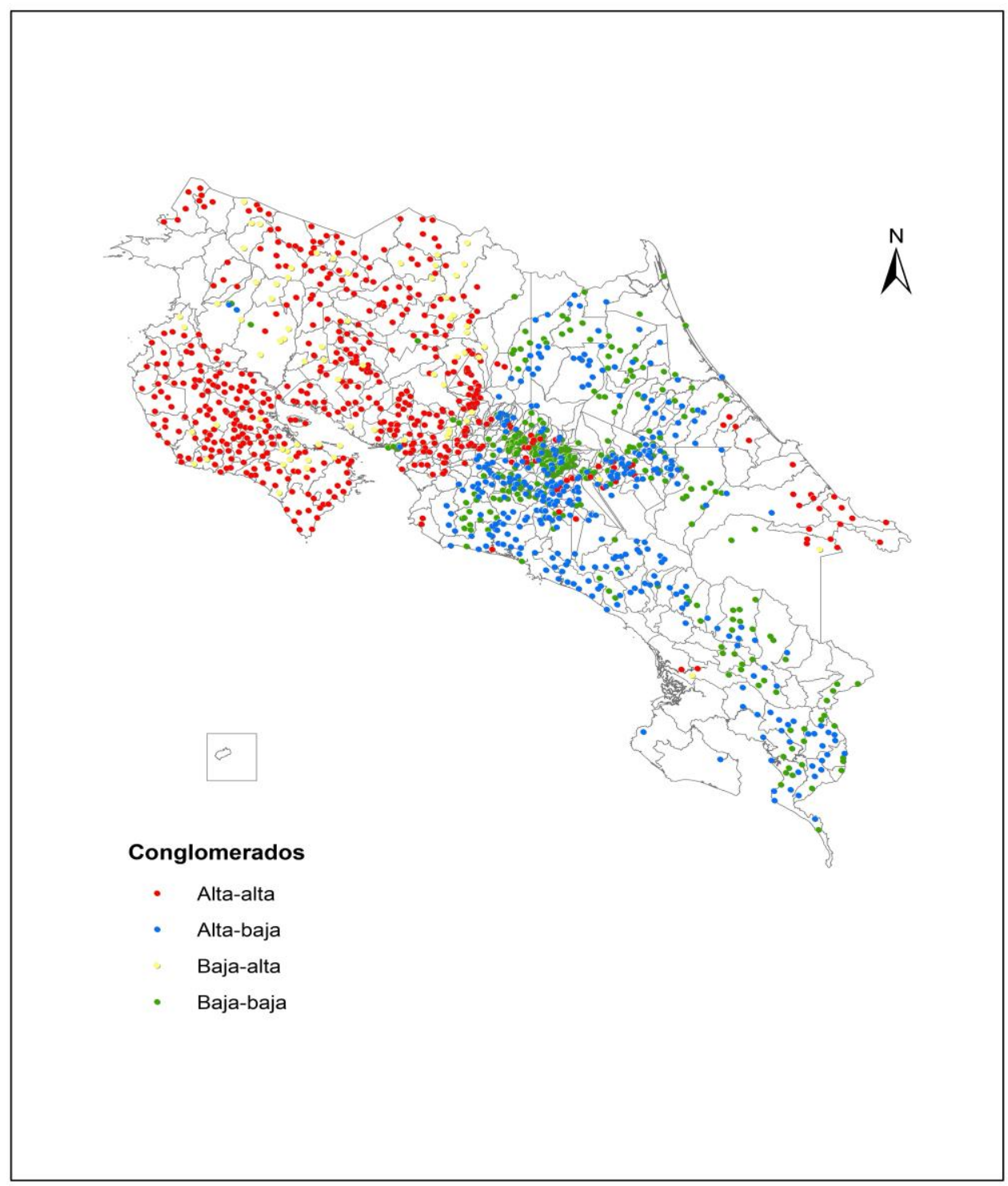

Figura 2. Conglomerados ${ }^{a /}$ de juntas y centros de votación con alta y baja participación electoral. 2016. Elaborado con base en los datos del Tribunal Supremo de Elecciones. a/ Puntos rojos: centros de alta votación rodeados de centros con niveles similares. Puntos verdes: centros de baja votación rodeados de centros con niveles similares. Puntos azules: centros de alta votación rodeados de centros de baja participación. Puntos amarillos: centros de baja participación rodeados de centros de alta participación. 


\section{DERECHO ELECTORAL}

\section{ANÁLISIS LONGITUDINAL DE LA PARTICIPACIÓN MUNICIPAL}

El análisis de la participación electoral territorial discutida arriba se complementa con un análisis de largo plazo de la asistencia a las urnas en las elecciones locales 2002-2016. Al respecto, la literatura especializada ha señalado que la participación electoral puede ser vista desde una perspectiva de hábito. De acuerdo con Plutzer (2002, p. 42) "existe un amplio consenso en la literatura especializada de que el comportamiento electoral es, al menos en parte, un hábito que se adquiere gradualmente". Los individuos aprenden el hábito de votar, o de abstenerse, basados en la experiencia de las primeras elecciones en las que tuvieron el derecho de sufragar (Franklin et al., 2004).

La lógica detrás de este supuesto es que la historia de voto de los ciudadanos es un poderoso predictor del comportamiento futuro. Brody y Sniderman (1977) señalaron que el comportamiento electoral previo predice la participación política actual, incluso luego de controlar por otras características individuales de los votantes y sus patrones de involucramiento en la política. A pesar de que es posible encontrar diferentes denominaciones en la literatura para caracterizar el hábito electoral ("voto consuetudinario", "inercia", "voto casual", "voto circunstancial"), lo cierto es que hay un acuerdo generalizado en el que se asume que la participación electoral es un hábito (Plutzer, 2002).

En síntesis, el haber sufragado en una elección aumenta la probabilidad de que un individuo acuda a las urnas en el futuro. Puntualmente, "si dos individuos poseen exactamente las mismas características, pero uno de ellos decide votar y el otro no lo hace, esas decisiones afectarán sus probabilidades de votar en elecciones futuras" (Denny y Doyle, 2009, p. 17). Es decir, "manteniendo los atributos individuales y el contexto como constantes, el simple hecho de votar incrementa las opciones de que este individuo siga haciendo lo mismo en próximas elecciones" (Green y Shachar, 2000, p. 562).

Tal y como ha sido establecido por diferentes expertos, "los individuos son criaturas de hábitos" (Danner et al., 2008, p. 245). Esta generalización considera el proceso de formación de un hábito como la consecuencia de un proceso repetitivo. "La repetición de un comportamiento en un contexto que no cambia, activa progresivamente una respuesta automática con el que se ejecuta el comportamiento cada vez que se tenga la posibilidad de hacerlo" (Lally et al., 2010, p. 999). En síntesis, una vez que un comportamiento es ejecutado repetidamente y se vuelve habitual, como el voto, este es guiado 


\section{DERECHO EIECTORAL}

por un proceso cognitivo en lugar de ser precedido por un elaborado proceso de decisión (Aarts et al., 1998).

Dado que en los cuatro comicios municipales celebrados (2002, 2006, 2010 y 2016) la participación de los costarricenses nunca ha superado el $35 \%$ del padrón, se ha afirmado de manera unánime e inequívoca que más de dos terceras partes de los ciudadanos con derecho a sufragar nunca han ejercido ese derecho. En estas circunstancias la escogencia de las autoridades locales se deja en manos de un reducido número de votantes.

En la práctica esto tiene serias repercusiones para la democracia local, pues el elevado ausentismo en las urnas margina a un amplio segmento del electorado cuyos intereses no son representados en las instancias políticas municipales. En el artículo se presentan los resultados de un análisis longitudinal de la participación de los costarricenses en los comicios municipales. Para ello, se construyó una base de datos que incluye al universo de votantes habilitados para sufragar en las elecciones locales de la primera década del siglo XXI (ver recuadro 2 ).

\section{Recuadro 2: panel de votantes en elecciones municipales}

En el marco del acuerdo de cooperación entre el Centro de Investigación y Estudios Políticos y el Tribunal Supremo de Elecciones, este último facilitó la información base para el análisis del comportamiento de los votantes costarricenses en los comicios municipales de 2002, 2006, 2010 y 2016 . Para cada una de esas elecciones se contaba con una base de datos que incluía información del votante (edad, género, distrito administrativo de residencia) así como su comportamiento (votante o abstencionista).

Se procedió a unir los archivos en una "base madre" usando como código común el número de cédula de cada votante. Dicho identificador es exhaustivo y exclusivo; es decir, todos los votantes poseen un número de cédula que, además, es diferente para cada persona. El siguiente paso fue transformar la base madre en un panel de electores con cuatro observaciones para cada individuo.

En una base de datos de panel cada individuo debe aparecer la misma cantidad de veces que observaciones en el tiempo, en este caso cuatro elecciones. El número total de observaciones es de 10979102 votantes. Esta cifra equivale a sumar los votantes empadronados en 2002, más los de 2006, 2010 y 2016 así como los que se incorporaron como nuevos votantes en 2006, 2010 y 2016. Asimismo, se excluyen los casos de personas que no estuvieron empadronadas en las elecciones respectivas.

Si bien es cierto en estos estudios una gran proporción de los estudiados se mantiene en el padrón a lo largo del tiempo, hay varias razones naturales por las que algunos individuos no aparecen empadronados en alguna o incluso en todas las 4 elecciones. Por ejemplo, algunos 


\section{DERECHO EIECTORAL}

votantes fallecen en el período entre elecciones; a otros se les pudo haber vencido la cédula por un período mayor a un año y no la renovaron (ambos motivos justifican su salida automática del padrón), estar fuera del país e incluso algunos pueden ser votantes que recién se incorporan al electorado. En síntesis, no todos los votantes han tenido la posibilidad de votar en la misma cantidad de elecciones, aunque una amplia mayoría de ellos ha podido votar en todas las cuatro elecciones estudiadas. La base de datos utilizada refleja las entradas y salidas normales de los electores en el padrón.

El principal hallazgo de este ejercicio es que en las elecciones municipales sufragan más votantes de lo que tradicionalmente se cree. La evidencia disponible demuestra que los "abstencionistas habituales"; es decir, aquellos que no votaron en ninguno de los cuatro comicios estudiados -ya sea por una decisión personal o porque no tuvieron derecho a hacerlo por no estar empadronados, por ejemplo- son menos de lo que se creía ${ }^{1}$. En efecto, 46 de cada 100 electores nunca acudieron a las urnas en comicios municipales.

En el otro extremo están los "votantes habituales" que, como su nombre lo dice, son aquellos que han votado todas las veces que han podido hacerlo. Este es un grupo reducido y asciende a 8 de cada 100 de los votantes registrados.

Esto quiere decir que los 54 de cada 100 electores restantes constituyen lo que podría denominarse como "votante volátil": 12 de ellos sufragaron en dos de los tres comicios efectuados, 15 de cada 100 participó la mitad de las veces ( 1 de 2 o 2 de 4 respectivamente), 8 de los 100 votaron el $75 \%$ de las veces en las que estaban habilitados para hacerlo y, finalmente, 3 de ellos acudieron a las urnas únicamente en una de las cuatro elecciones a las que tuvieron o derecho.

La figura 3 ilustra la distribución del comportamiento de los casi 11 millones de costarricenses que han tenido derecho a participar en uno o más de los cuatro comicios municipales estudiados.

\footnotetext{
${ }^{1}$ Para facilitar la comprensión de los resultados en este apartado se asume que la totalidad de observaciones del panel (más de 10 millones de observaciones) es igual a 100.
} 


\section{DERECHO ELECTORAL}

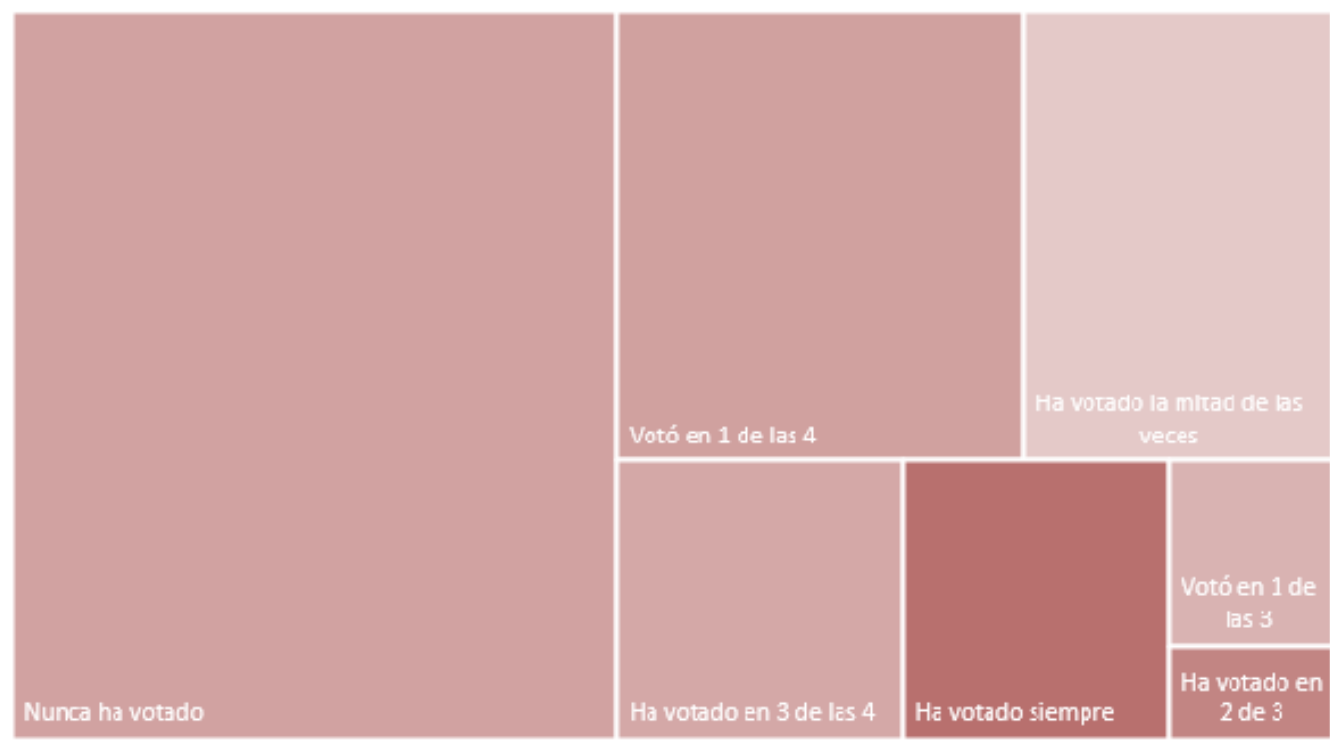

Figura 3. Universo de votantes y proporción de veces que han votado en las elecciones municipales 2002-2016.

Los "votantes volátiles", que representan un poco más de la mitad de los electores (54 de cada 100), plantean un dilema para la participación en elecciones locales en el futuro. Por un lado, podría especularse que este grupo podría votar en futuras elecciones dado que ya lo hicieron al menos una vez en el pasado. Esto podría estimular la concurrencia a las urnas y producir un incremento de cara al futuro. En caso de que así suceda se trataría de votantes que reconectan con el sistema político local, pues su ausencia, transitoria, no acarrea una salida permanente del sistema democrático.

Por otro lado, si en lugar de acudir a las urnas deciden abandonarlas indefinidamente, el panorama para la participación es menos promisorio y dependería en mayor medida del comportamiento de los nuevos votantes que se incorporen en futuras elecciones. Independientemente de lo que pase con los "votantes volátiles", no podría descartarse la posibilidad de que entre los "abstencionistas habituales", algunos acudan a las urnas en 2020 o 2024. Una muestra representativa de 300 votantes ilustra la distribución del comportamiento de las y los costarricenses en tres de los comicios municipales analizados, como se observa en la figura 4. 


\section{DERECHO ELECTORAL}

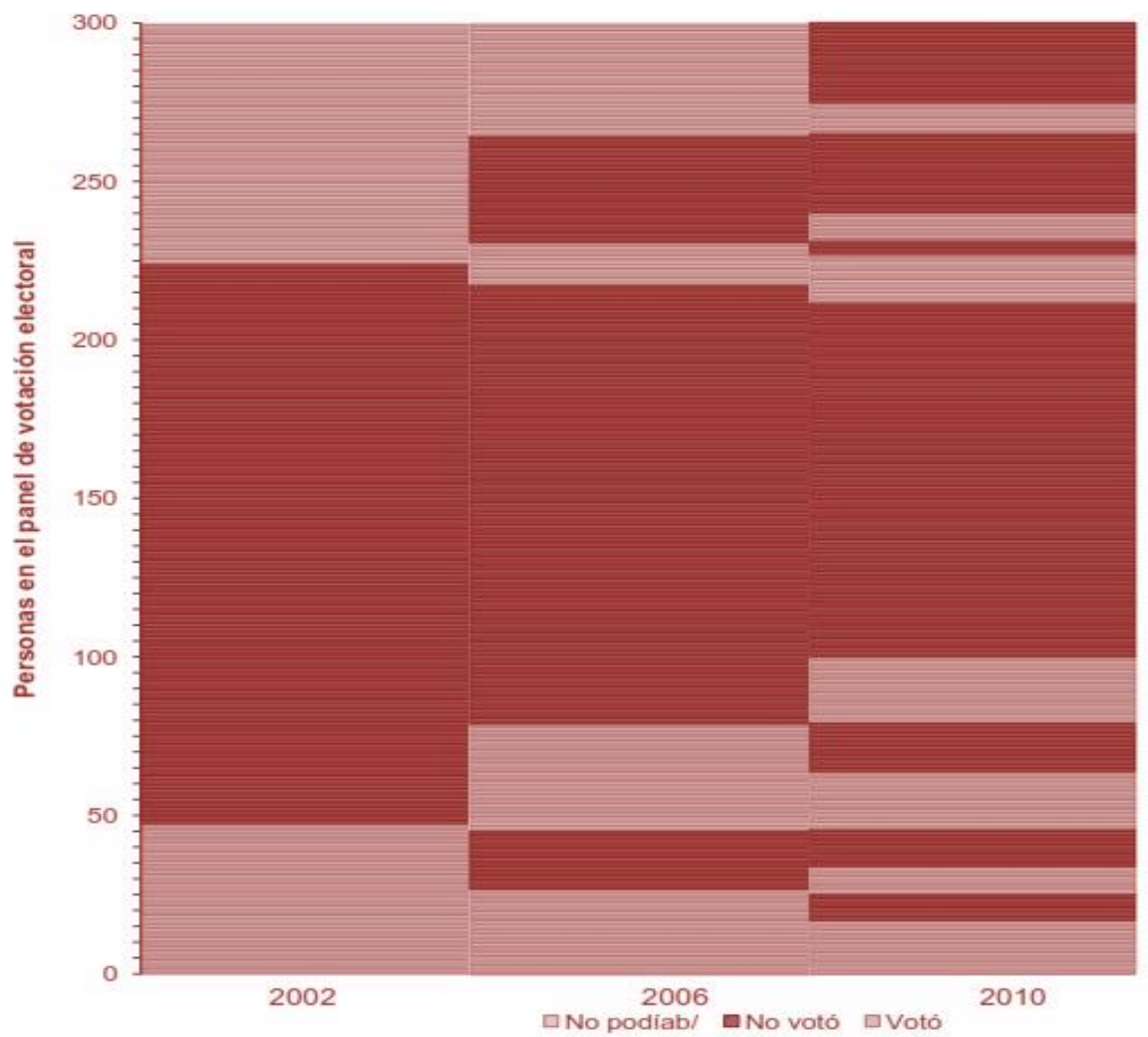

Figura 4. Comportamiento electoral en elecciones municipales ${ }^{\mathrm{a} /}$. 2002, 2006 y 2010. Tomado de Programa Estado de la Nación, 2015

a/ Cada individuo es una línea. Un cambio en el color de la línea indica un cambio en el comportamiento electoral.

b/ La opción "No podía" significa que el votante no se encontraba empadronado en el momento de la elección.

Un segundo hallazgo es que las características sociodemográficas tienen un peso importante en la explicación del comportamiento electoral a nivel local, según un análisis de dos modelos de regresión estadística. Para efectos analíticos se emplearon dos modelos estadísticos. Se parte de un modelo básico (modelo 1), al cual se le agregan dimensiones y variables. En este se incluye un conjunto elemental de indicadores sociodemográficos y se explora su asociación con la participación en las elecciones municipales del 2016. El modelo 2 incorpora variables políticas tales como el partido ganador en las elecciones presidenciales y la agrupación que controla la mayoría del concejo municipal. Se usa como base el PLN por ser el partido dominante, hasta ahora, a escala local. 


\section{DERECHO EIECTORAL}

Según estos modelos, en concreto, ser mujer, estar casado y ser mayor de edad son factores asociados a una mayor participación en los comicios (figura 5). Además, un elector que vive en un distrito urbano tiene una propensión a votar significativamente menor que la de un residente de un distrito rural.

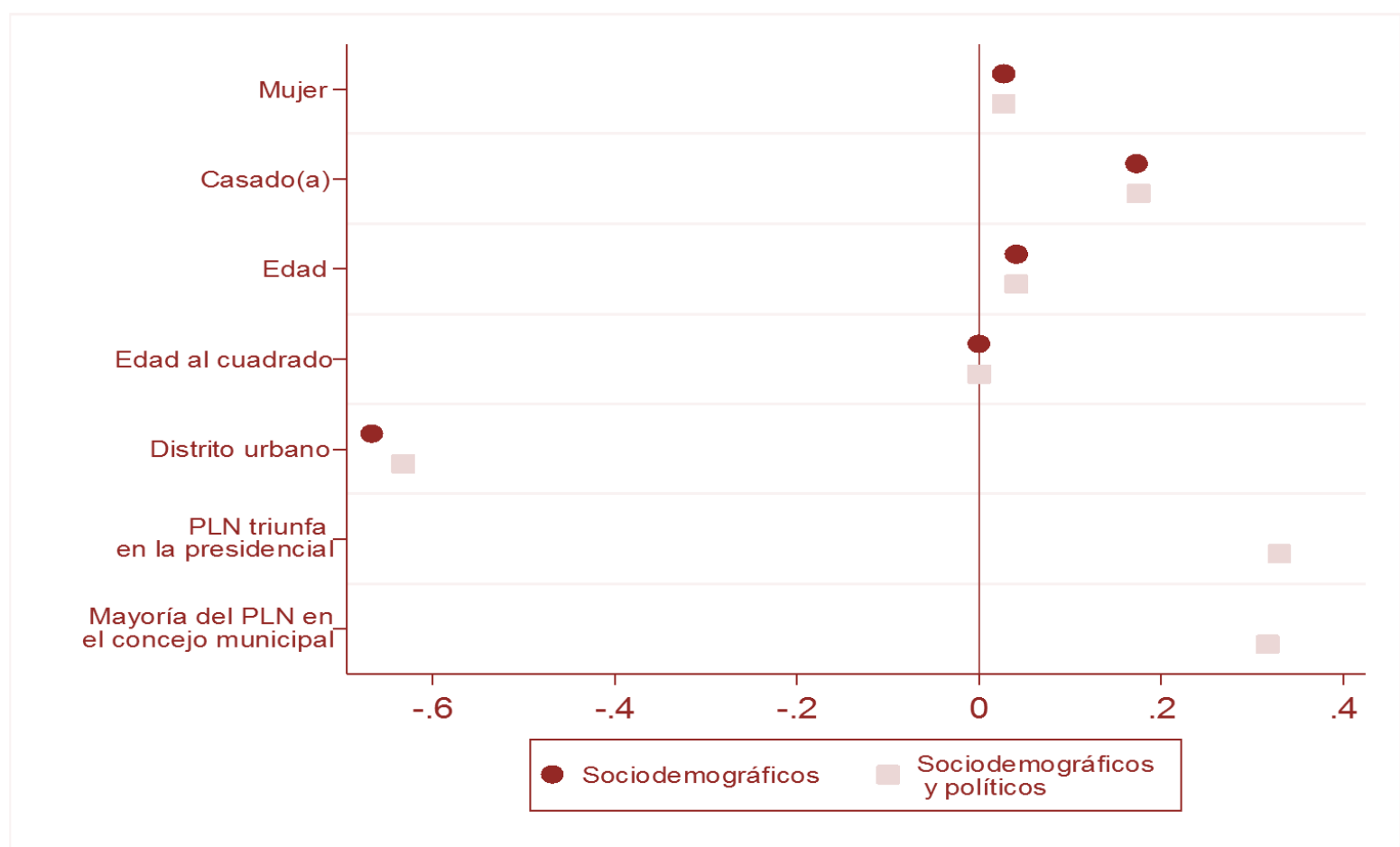

Figura 5. Factores asociados a la participación electoral en comicios municipales (modelos de regresión logísticaa/). Tomado de Programa Estado de la Nación, 2015

a/ El gráfico muestra los resultados de dos regresiones logísticas en las que la variable dependiente es la participación en las elecciones municipales (votó: sí o no) e incluye una línea vertical en el número "0". Cuando el coeficiente estimado de una variable se ubica a la izquierda de la línea, indica que esta tiene una relación negativa con la variable dependiente; cuando el coeficiente se ubica a la derecha, significa lo contrario. Se puede tener un $95 \%$ de confianza de que esa relación es estadísticamente significativa cuando el círculo o el cuadrado no atraviesan la línea vertical.

Un tercer y último hallazgo muestra que, si bien las características sociodemográficas ayudan a explicar la concurrencia a las urnas, los factores políticos tienen un poder predictivo incluso mayor. En este sentido, el análisis de regresión multivariado reveló que, cuando en un cantón un 


\section{DERECHO EIECTORAL}

mismo partido - por ejemplo el PLN2- triunfa en los comicios presidenciales y a la vez obtiene una mayoría en el concejo municipal, se incrementa la participación ciudadana. Una posible explicación de este fenómeno es que los votantes se movilizan en la medida en que perciben el predominio de una agrupación en los niveles nacional y municipal.

De no existir esa hegemonía, los individuos estarían más inclinados a abstenerse. No obstante, esta es una hipótesis que debe ser evaluada con más datos, para lo cual se requiere una serie de tiempo mayor. En síntesis, la mejor combinación para la participación en elecciones municipales es la hegemonía de un mismo partido en los contextos nacional y local.

\section{CONCLUSiones}

Este artículo resume los principales hallazgos de los patrones macro y micro de la participación de los costarricenses en elecciones municipales recientes. Costa Rica ha sido tradicionalmente un país centralista desde el punto de vista político-administrativo. Esta condición tiene profundas repercusiones en la forma en la que los ciudadanos se relacionan con sus Gobiernos y autoridades en sus comunidades.

Los principales hallazgos son tres. En primer lugar, el análisis territorial de la participación electoral muestra un país dividido en dos: los ciudadanos del este del país concurren menos a las urnas que los que residen en el oeste del territorio. Las localidades de mayor participación se concentran en Guanacaste, parte de Puntarenas y Alajuela, y pequeños conglomerados en Limón, Cartago, Heredia y San José o incluso en la zona sur. Mientras que las regiones de baja participación se ubican, fundamentalmente, en el centro del territorio y en zonas costeras y limítrofes. Este patrón es marcadamente distinto del identificado para las elecciones nacionales en el que predomina la división centro y periferia, de alta y baja participación respectivamente (PEN, 2017).

En segundo lugar, un análisis longitudinal identificó que los votantes habituales en elecciones locales rondan el $10 \%$ de los habilitados para votar y

\footnotetext{
${ }^{2}$ El PLN se utilizó como categoría base para el análisis por dos razones: I) fue el partido que ganó dos de las tres elecciones presidenciales entre 2002 y 2010, y II) fue el partido con mayor cantidad de alcaldes electos en dos de los tres comicios municipales.
} 


\section{DERECHO ELECTORAL}

que 4 de cada 10 votantes sufragaron, al menos en una de las tres elecciones celebradas entre 2002 y 2016.

Por último, un análisis de regresión logística concluye que las características sociodemográficas tienen un peso importante en la explicación del comportamiento electoral a nivel local. En concreto, ser mujer, estar casado y tener más edad son factores asociados a la participación en dichos comicios. Además, un elector que vive en un distrito urbano tiene una propensión a votar significativamente menor que la de un residente de un distrito rural.

No obstante, si bien las características sociodemográficas ayudan a explicar la concurrencia a las urnas, los factores políticos tienen un poder predictivo incluso mayor. En este sentido, la mejor combinación para la participación en elecciones municipales es la hegemonía de un mismo partido en los contextos nacional y local.

\section{REFERENCIAS BIBLIOGRÁFICAS}

Aarts, H.; Verplanken, B. y Knippenberg, A. (1998). Predicting Behavior From Actions in the Past: Repeated Decision Making or a Matter of Habit. Journal of Applied Social Psychology 28(15), 1355-1374.

Anduiza, E. y Bosch, A. (2004). Comportamiento político y electoral. Barcelona: Editorial Ariel S.A.

Alfaro, R. (2002). Elecciones municipales y debilitamiento del respaldo electoral bipartidista en Costa Rica. Período 1986-2002. Anuario de Estudios Centroamericano, 28(1-2), 89-135.

Alfaro, R. (2006): Elecciones nacionales 2006 en Costa Rica y la recomposición del sistema de partidos políticos. Revista de Ciencia Política, 26(1), 125-137.

Alfaro, R. (2008). Elecciones de alcaldes municipales 2006 en Costa Rica: muchos los llamados, pocos los concurridos. Revista Centroamericana de Ciencias Sociales, 5(1), 169-203.

Alfaro, R. (2009). El régimen municipal costarricense a inicios del siglo XXI, Serie Cuadernos para el Ciudadano, vol. 8. San José, Costa Rica: Editorial de la Universidad de Costa Rica. 


\section{DERECHO EIECTORAL}

Alfaro, R. y Gómez, S. (2014). Costa Rica: Elecciones en el contexto político más adverso arrojan la mayor fragmentación partidaria en 60 años. Revista de Ciencia Política, 34(1), 125-144.

Blais, A., Gidengil, E. y Nevitte, N. (2004). Where does turnout decline come from? European Journal of Political Research, 43(2), 221-236.

Brody, R. A. y Sniderman, P. M. (1977). From Life Space to Polling Place: The Relevance of Personal Concerns for Voting Behavior. British Journal of Political Science, 7(03), 337-360.

Costa Rica. Asamblea Legislativa (1998). Ley 7794: Código Municipal. San José: Asamblea Legislativa.

Costa Rica. Asamblea Legislativa (2007). Ley 8611: Modificación de los artículos 14, 19 y 20 del Código Municipal, Ley 7794. San José: Asamblea Legislativa.

Costa Rica. Contraloría General de la República. (2015). Resultados del índice de gestión municipal 2014. División de Fiscalización Operativa y Evaluativa. San José: Contraloría General de la República.

Danner, U. N.; Aarts, H. y Vries, N. K. (2008). Habit vs. intention in the prediction of future behaviour: The role of frequency, context stability and mental accessibility of past behaviour. British Journal of Social Psychology 47(2), 245265.

Denny, K. y Doyle, O. (2009). Does Voting History Matter? Analysing Persistence in Turnout. American Journal of Political Science 53(1), 17-35.

Delgado, I. (May.-Ago., 1998). El comportamiento electoral en los municipios rurales: una aproximación desde las elecciones municipales de 1995. Agricultura y Sociedad, (86), 13-31.

Franklin, M. N. (2004). Voter turnout and the dynamics of electoral competition in established democracies since 1945. Cambridge: Cambridge University Press.

Franklin, M. N.; Lyons, P. y Marsh, M. (2004). Generational Basis of Turnout Decline in Established Democracies. Acta Política 39(2), 115-151.

Flickinger, R. S. y Studlar, D. T. (1992). The disappearing voters? Exploring declining turnout in Western European elections. West European Politics 15(2), 1-16. 


\section{DERECHO ELECTORAL}

Green, D. P. y Shachar, R. (2000). Habit Formation and Political Behaviour: Evidence of Consuetude in Voter Turnout. British Journal of Political Science 30(4), 561573.

Heath, A. et al. (1999). Between first and second order: A comparison of voting behaviour in European and local elections in Britain. European Journal of Political Research, 35(3), 389-414.

Holbrook, T. y Weinschenk, A. (2013). Campaigns, mobilization, and turnout in mayoral elections. Political Research Quarterly, 67(1), 42-55.

Jones, M. y Mainwaring, S. (2003): The nationalization of parties and party systems: an empirical measure and an application to the Americas. Party Politics. 9(2), 139-166.

Lally, P.; Jaarsveld, C.; y Potts, H. y Wardle, J. (2010). How are habits formed: Modelling habit formation in the real world. European Journal of Social Psychology 40(6), 998-1009.

Lehoucq, F. (1997). Lucha Electoral y Sistema Político en Costa Rica, 1948-1998. Colección Pasado y Presente. San José, Costa Rica: Editorial Porvenir.

Lewis-Beck, M. S. (2008). The American Voter Revisited. Ann Arbor: University of Michigan Press.

Mainwaring, S. (1993). Presidentialism, Multipartism, and Democracy: The Difficult Combination. Comparative Political Studies 26(2), 198-228.

Matsusaka, J. G. y Palda, F. (1999). Voter turnout: How much can we explain? Public Choice 98(3), 431-446.

Molina, J. (2007). Sistemas electorales subnacionales. En Nohlen, D. et al. (compiladores) Tratado de derecho electoral comparado de América Latina, pp. 334-370. México: Fondo de Cultura Económica.

Müller, W. (1988). Irrelevant elections: the quality of local democracy in Britain. Oxford University Press, USA.

Navia, P. (2003). Comportamiento electoral comunal 1996-2001 con miras a las municipales del 2004. Recuperado de http://www.icso.cl/archivos/comportamiento-electoral-comunal.pdf

Nie, N. H.; Verba, S. y Petrocik, J. R. (1976). The changing American voter. Cambridge: Harvard University Press. 


\section{DERECHO EIECTORAL}

Powell, G. B., Jr. (1986). American Voter Turnout in Comparative Perspective. The American Political Science Review 80(1). 17-43.

Programa Estado de la Nación. (2001). Auditoría Ciudadana Sobre la Calidad de la Democracia. San José. C.R.: Proyecto Estado de la Nación.

Programa Estado de la Nación (2010). Decimosexto informe Estado de la Nación. San José. C.R.: Proyecto Estado de la Nación.

Programa Estado de la Nación (2011). Decimoséptimo informe Estado de la Nación. San José. C.R.: Proyecto Estado de la Nación.

Programa Estado de la Nación (2015). Vigesimoprimer informe Estado de la Nación. San José. C.R.: Proyecto Estado de la Nación.

Programa Estado de la Nación (2017). Vigesimotercer informe Estado de la Nación. San José. C.R.: Proyecto Estado de la Nación.

Plutzer, E. (2002). Becoming a Habitual Voter: Inertia, Resources, and Growth in Young Adulthood. American Political Science Review 96(01), 41-56.

Raventós, C. et al. (2005). Abstencionistas en Costa Rica: ¿Quiénes son y por qué no votan? San José, Costa Rica: Editorial de la Universidad de Costa Rica.

Sánchez, F. (2001). Sistema electoral y partidos políticos: incentivos hacia el bipartidismo en Costa Rica. Anuario de Estudios Centroamericanos, 27(1), 133168.

Sánchez, F. (2004). Desalineamiento electoral en Costa Rica. Revista de Ciencias Sociales, 4(98), 29-56.

Teixeira, R. A. (1987). Why Americans don't vote : turnout decline in the United States, 1960-1984. New York: Greenwood Press.

Tobler, W. R. (1970). A computer model simulation of urban growth in the Detroit region. Economic Geography 46(2), 234-240.

Tribunal Supremo de Elecciones (2008): Resolución 405-E8-2008 las siete horas veinte minutos del ocho de febrero.

Trounstine, J. (2013). Turnout and Incumbency in Local Elections. Urban Affairs Review, 49(2), 167-189. 


\section{DERECHO ELECTORAL}

Urcuyo, C. (2003). Reforma Política y Gobernabilidad. San José, Costa Rica: Editorial Juricentro.

Vargas, J. (2007). Costa Rica: Fin de una era Política. Revista de Ciencia Política 27, 113-28.

Wattenberg, M. (1998). Turnout Decline in the U.S. and other Advanced Industrial Democracies. Irvine: University of California Irvine, School of Social Sciences, Center for the Study of Democracy. 